\title{
The NBRDETP: Neighbor Node Detection and Prediction Technique for Energy Efficient Routing with Leach Protocol in WSN
}

\author{
Navjot Kaur ${ }^{1}$, Jatinder Kumar ${ }^{2}$ \\ Department of Computer Science \& Engg, SSCET, Manawala, Amritsar ${ }^{1}$ \\ Asst Professor Department of Computer Science \& Engg SSCET, Manawala, Amritsar ${ }^{2}$
}

\begin{abstract}
In this paper, cluster head and vice cluster head selection is discussed and a method of energy efficient routing is presented based on both NBRDETP and V-LEACH protocol. Performance comparison with existing leach protocol shows proposed protocol provides better performance to minimize energy dissipation in the transmission and increases the life time of the wireless sensor networks, also other comparative performance metrics like End to End delay, data transmitted and total energy consumed shows proposed protocol provides better performance in comparison to existing leach protocol.
\end{abstract}

Keywords: PSO, NBRDETP, WSN, Vleach.

\section{INTRODUCTION}

A wireless sensor network (WSN) is a wireless network using sensors to considerately monitor physical or environmental condition [4]. A wireless sensor network consists of three basic components: the sensor nodes, power source and a central controller. The sensor nodes in turn are comprised of sensors and transceiver module [5, 6]. Sensors are used to determine the changes to physical environment like pressure, humidity, sound, vibration and changes to the health of person like blood pressure, stress and heartbeat $[6,8]$. The transceiver module transmits the data from sensor to the central controller through a wireless medium. The power source provides the power needed for the sensor nodes and this power is mostly regenerative. The central controller unit performs all the computations for the sensor networks. The controller receives the input from all sensors and processes simultaneously to make the essential decisions. [5, 6]

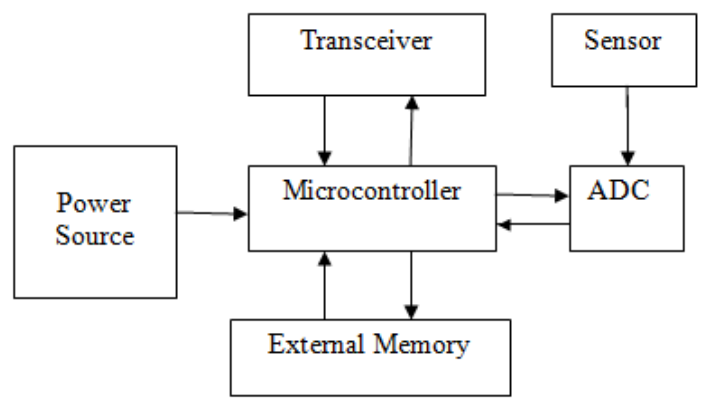

Fig. 1: Components of Sensor Node

The sensor nodes are able or capable for performing the process on the gathered data and after processing communication with other connected nodes in the networks. The data will be sent from the source node to the sink node. Sink node always collect the data from other connected nodes from the network. [7]

A WSN usually consists of a large number of inexpensive, low-power, and multifunctional wireless sensor nodes, with sensing, wireless communications and computation capabilities [7]. The wireless sensor nodes communicate over short distance through a wireless medium and collaborate to achieve a common action, for example, environment monitoring, military surveillance, health care, structural monitoring, military, forest surveillance, habitat monitoring and agriculture. $[9,10]$

One of the major issues of wireless sensor network is power consumption. To solve this problem two methods are defined. The first method is to establish aggregation points. Then there is reduction on the total number of messages 
Vol. 6, Issue 6, June 2017

exchanged between sensor nodes and saves some amount of energy. Usually aggregation points are common nodes that receive data from neighbouring nodes, carry out processing and then forward the filtered data to next hop. [3]

The most important purpose of data aggregation is to increase the network life span by reducing the utilization of sensor nodes such as battery energy and bandwidth. Data aggregation is defined as the process of collecting the data from multiple sensor nodes to remove unnecessary transmission and provide amalgamated information to the base station [11]. Data aggregation generally involves the data in collective form from multiple sensor nodes at midway nodes and transmission of the aggregated data to the sink node (base station). [2]

\section{RELATED WORK}

K. Hari Krishna proposed A wireless sensor network consists of many wireless nodes forming a network which are used to monitor certain physical or environmental conditions, such as humidity, temperature, sound etc. Some of the popular applications of sensor network are area monitoring, environment monitoring (such as pollution monitoring), and industrial and machine health monitoring, waste water monitoring and military surveillance. Topology control in WSNs is a technique of defining the connections between nodes in order to reduce the interference between them, save energy and extend network lifetime..

Pardeep Kaur proposed [3] Wireless sensor network is emerging field because of its wide applications in various fields and least cost. A wireless sensor network is a group of small sensor nodes which communicate through radio interface. These sensor nodes are composed of sensing, computation, communication and power as four basic working units. But limited energy, communication capability, storage and bandwidth are the main resource constraints. Our survey is based on various aspects of wireless sensor networks..

Deepak Ganesan proposed [4] Previously proposed sensor network data dissemination schemes require periodic low rate flooding of data in order to allow recovery from failure. Consider constructing two kinds of multipath to enable energy efficient recovery from failure of the shortest path between source and sink. Disjoint multipath has been studied in the literature. a novel braided multipath scheme, which results in several partially disjoint multipath schemes. that braided multipath are a viable[12] alternative for energy-efficient recovery from isolated and patterned failures. Node level and a particular node broadcasts the data to all other nodes by acting as cluster head .In MASP, the mapping between sensor nodes and sub sinks optimized to maximize the amount of data collected by mobile sinks and the energy consumption is balanced.

\section{PROPOSED METHOD}

The proposed protocol NBRDETP is a proactive routing protocol, in which all the paths are discovered prior to its requirement. This NBRDETP approach is suitable for the static network. NBRDETP is a cluster-based routing protocol that requires the route from the cluster head to the base station. The base station is responsible for computing the routing path and monitoring the energy level of each sensor node in the network. It consists of four phases: neighbour discovery and topology construction, cluster head selection and cluster formation, data transmission, and re-clustering and rerouting. The base station initiates neighbour discovery[11] phase after the deployment of sensor nodes. Here each sensor node will broadcast NBRDETP packet once. At the end of the neighbour discovery phase, each node has the information about their neighbours. Each node broadcasts the NBRDETP control packet as shown in Figure 3.1. The NBRDETP packet consists of sender id. Whenever a node receives the NBRDETP packet, it does the following operations:

1. Checks the neighbour list for the existence of the sender node id. If the sender id is not available in the neighbour list, then add it, else drops the packet.

2. If NBRDETP Sent is false, then recipient node makes NBRDETP Sent as true and broadcasts the NBRDETP packet.

ALOGRITM: Senor Node Route Path

1: for time $=1$ to simulation time

2: for $\mathrm{i}=1: \mathrm{N}$, where $\mathrm{N}$ the number of Model Sensory nodes in network

3: for neighbour node Analyse the effect of less neighbour hop count

4 Find normal pattern broken point

5: if normal pattern broken (i) arises

6: Apply NBRDETP neighbour node technique for optimal route transmission

7:else

8:network with normal nodes and efficient Path 
9: endif

10: end

11: end

12: end

Flow Chart for Proposed technique NBRDETP

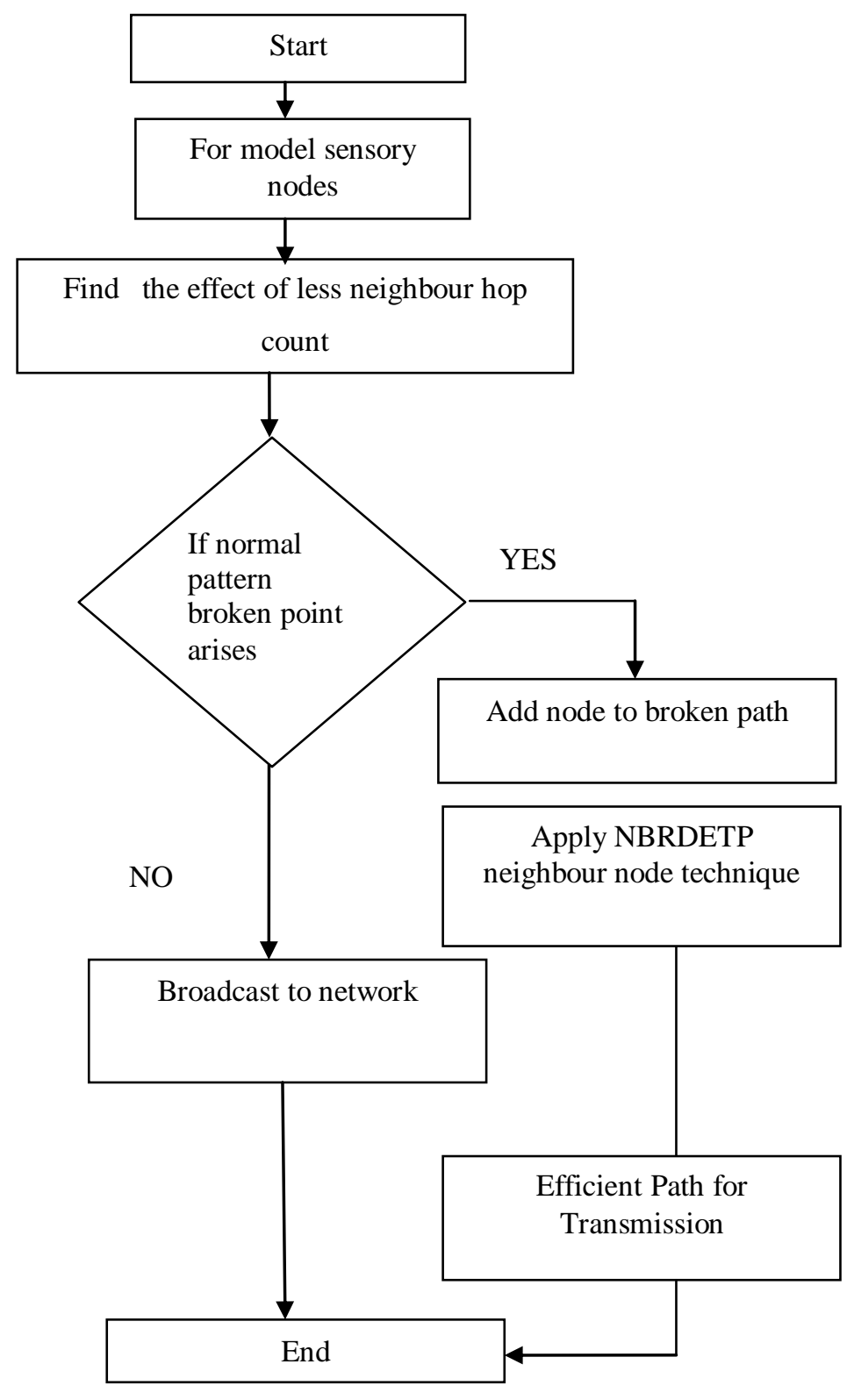

IV RESULT ANALYSIS

\begin{tabular}{|c|c|c|}
\hline S. No. & Parameter & Value(s) \\
\hline 1 & Simulator used & NS 2.35 \\
\hline 2 & Simulation Time & 10 Secs \\
\hline 3 & Simulation Area & 1000 X 1000 \\
\hline 4 & MAC & 802.11 \\
\hline 5 & Number of nodes & 50 \\
\hline 6 & Speed of Nodes & 2 to 16 (m/sec) \\
\hline 7 & Mobility Model & Random Waypoint \\
\hline
\end{tabular}


Vol. 6, Issue 6, June 2017

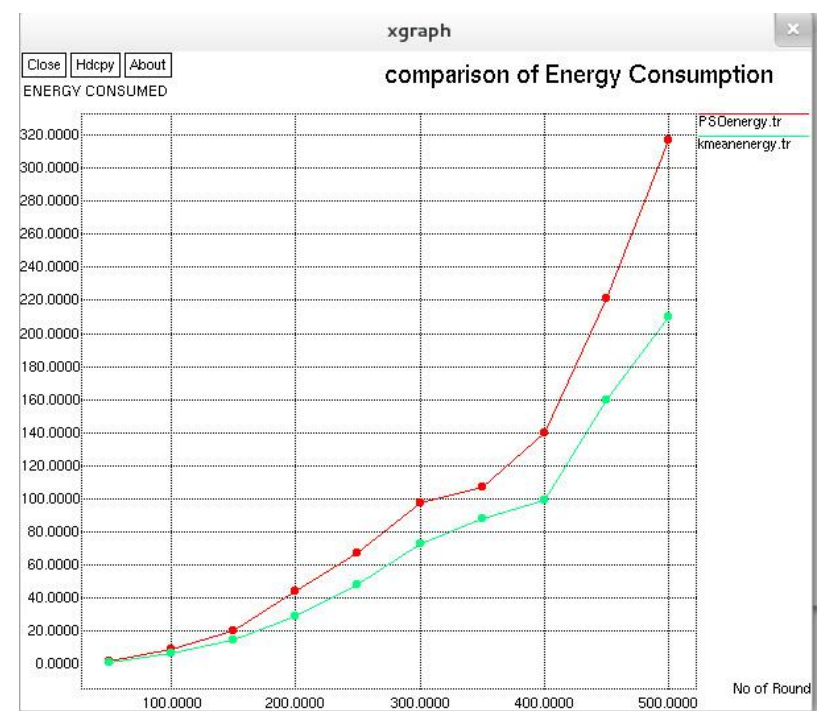

Fig1 comparison of Energy Consumption

Table1comparison of Energy Consumption

\begin{tabular}{|c|c|c|}
\hline No of Rounds & NBRDETP & PSO \\
\hline 50 & 49 & 53 \\
\hline 100 & 52 & 56 \\
\hline 150 & 54 & 59 \\
\hline 200 & 64 & 68 \\
\hline 250 & 69 & 70 \\
\hline 300 & 71 & 72 \\
\hline 350 & 72 & 74 \\
\hline 400 & 75 & 77 \\
\hline 450 & 80 & 82 \\
\hline 500 & 97 & 105 \\
\hline
\end{tabular}

The analysis of Energy consumption with previous technique for finding irregular path are shown in Fig1 the results in Table1 shows that Energy consumption using NBRDETP neighbour is low as compared to previous technique PSO with increase in no of nodes .which shows our technique NBRDETP neighbour has better results as compared to previous technique PSO in terms of energy Consumption

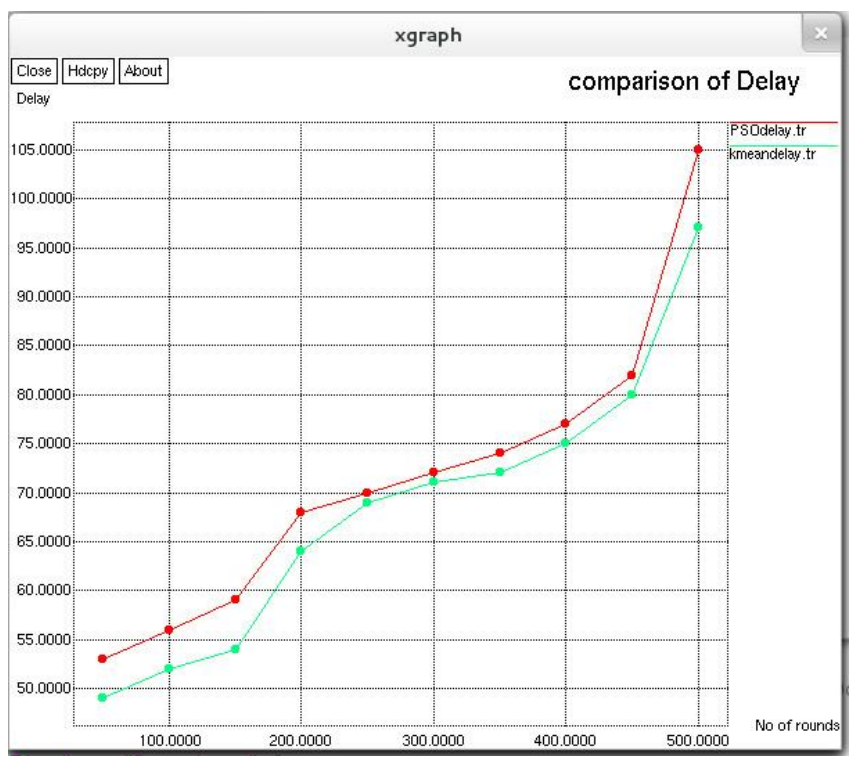

Fig 2 Comparison of Data Delay 
Vol. 6, Issue 6, June 2017

Table2comparison of Data Delay

\begin{tabular}{|c|c|c|}
\hline No of Rounds & NBRDETP & PSO \\
\hline 50 & 49 & 53 \\
\hline 100 & 52 & 56 \\
\hline 150 & 54 & 59 \\
\hline 200 & 64 & 68 \\
\hline 250 & 69 & 70 \\
\hline 300 & 71 & 72 \\
\hline 350 & 72 & 74 \\
\hline 400 & 75 & 77 \\
\hline 450 & 80 & 82 \\
\hline 500 & 97 & 105 \\
\hline
\end{tabular}

The Data Delay Metric is used for finding effectiveness of NBRDETP with respect to number movable sensor nodes. The Fig2 and Table2 values illustrate the relative measure of data Delay using the proposed NBRDETP neighbor and PSO. an decreased difference in irregular path in Proposed Technique NBRDETP neighbour specifies more efficient result in data collection as compared to Previous technique PSO

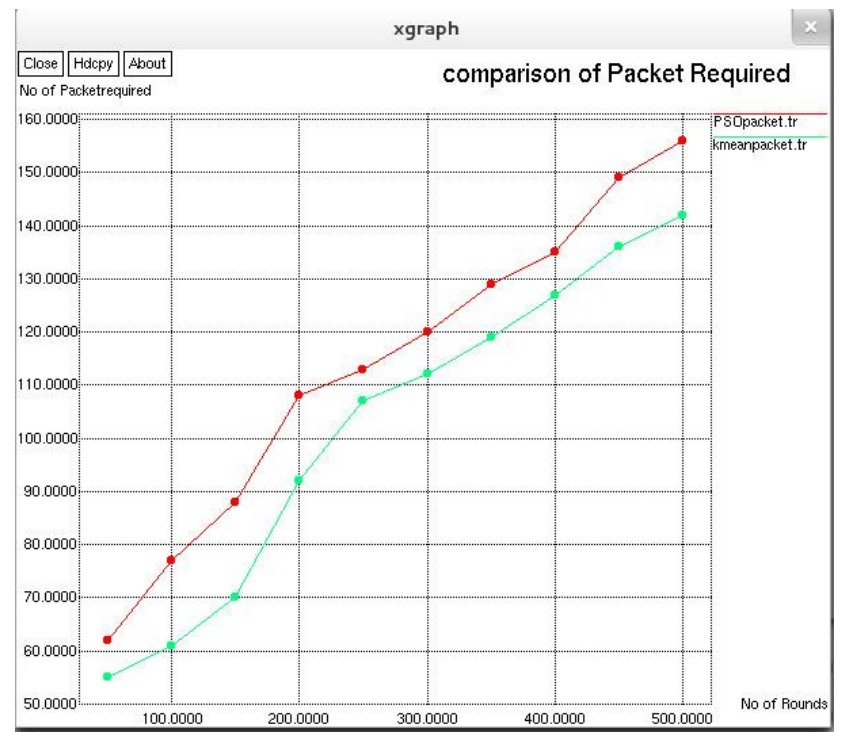

Fig 3 Comparison of No Packet required

Table3comparison of Packet required

\begin{tabular}{|c|c|c|}
\hline No of Rounds & NBRDETP & PSO \\
\hline 50 & 55 & 62 \\
\hline 100 & 61 & 77 \\
\hline 150 & 70 & 88 \\
\hline 200 & 92 & 108 \\
\hline 250 & 107 & 113 \\
\hline 300 & 112 & 120 \\
\hline 350 & 119 & 129 \\
\hline 400 & 127 & 135 \\
\hline 450 & 136 & 149 \\
\hline 500 & 142 & 156 \\
\hline
\end{tabular}

The No of Packet is used for finding effectiveness of NBRDETP with respect to number movable sensor nodes. The Fig3 and Table3 values illustrate the relative measure of Packet Required using the proposed NBRDETP neighbor and PSO. an decreased difference in irregular path in Proposed Technique NBRDETP neighbour specifies more efficient result in data collection as compared to Previous technique PSO 


\section{CONCLUSION}

In this paper, presented on NBRDETP energy efficient routing protocol for wireless Sensor networks. We have analysed performance metrics Data Transmitted, Consumed energy, End to End delay for comparison, performance comparison shows proposed protocol provides better performance. Energy consumed in proposed protocol is less in comparison to existing protocol, delay time in proposed protocol is less in comparison to existing protocol, also no of packets required to send same amount of data is less in proposed protocol in comparison to existing protocol

\section{REFERENCES}

[1] Alka Singh, Shubhangi Rathkanthiwar and Sandeep Kakde, "Energy Efficient Routing of WSN using ParticleSwarm Optimization and VLeach Protocol”, Volume 79, April 6-8, 2016, Pages 2078-2082.

[2] K. Hari Krishna , Y. Suresh Babu.Dr And Tapas Kumar. Dr, "Wireless Sensor Network Topology Control using Clustering " ,Volume 79, 2016, Pages 893-902.

[3] Pardeep Kaur and Vinay Bhardwaj , "A survey on sensor networks," International Journal of Advanced Research in Computer Science and Software Engineering, vol. 5, ISSN: 2277 128X, 2015, pp. 988-994.

[4] D. Ganesan, R. Govindan, S. Shenker, and D. Estrin, "Highly-resilient, energy-efficient multipath routing in wireless sensor networks," vol. 2, pp. 1-13, 2015 .

[5] Te-Li Wang, Jung-Chun Kao, and Si An Ciou, "A traffic-adaptive energy-efficient MAC protocol for wireless sensor networks", vol.5, 2014.

[6] Santar Pal Singh And S.C. Sharma, "A Survey on Cluster Based Routing Protocols in Wireless Sensor Networks", Volume 45, 2015, Pages 687-695.

[7] Amit Sarkar and T. Senthil Murugan, "Routing protocols for wireless sensor networks: What the literature says" ,Volume 55, 2016, Pages 3173-3183.

[8] T. He, J. Stankovic, C. Lu, and T. Abdelzaher, "Speed: A stateless protocol for real-time communication in sensor networks", Vol.5, pp. 1-13, 2016.

[9] Chenyang Lu Brian M. Blum Tarek F. Abdelzaher John A. Stankovic Tian He, "Rap: A real-time communication architecture for large-scale wireless sensor networks," in IEEE Real-Time and Embedded Technology and Applications Symposium, vol.5, 2016.

[10] Sukhkirandeep Kaur and Roohie Naaz Mir, "Clustering in Wireless Sensor Networks- A Survey", I. J. Computer Network and Information Security, vol.6,pp. 38-51, 2016.

[11] Gurbinder Singh Brar, Shalli Rani, Vinay Chopra, Rahul Malhotra, Houbing Songand Syed Hassan Ahmed, "Energy Efficient Direction-Based PDORP Routing Protocol for WSN", vol.4,pp.3182-3194, 2016

[12] Abdul Razaque, Musbah Abdulgader and Chaitrali Joshi, "P-LEACH: Energy Efficient Routing Protocol for Wireless Sensor Networks, vol.5, pp.5-9, 2016. 